Global Journal of Pure and Applied Mathematics.

ISSN 0973-1768 Volume 13, Number 10 (2017), pp. 7573-7600

(C) Research India Publications

https://dx.doi.org/10.37622/GJPAM/13.10.2017.7573-7600

\title{
Simulated Annealing Conditions to Generate a New Population in Genetic Algorithm
}

\author{
G.S. Biju \\ Department of Mathematics, \\ College of Engineering, \\ Thiruvananthapuram, Kerala, India, 695016. \\ A.K. Anilkumar \\ Vikram Sarabhai Space Centre, \\ Indian Space Research Organisation, \\ Thiruvananthapuran, India.
}

\begin{abstract}
Genetic algorithms and simulated annealing are two important iterative algorithms widely used in combinatorial optimizations. While genetic algorithm is a probabilistic algorithm inspired in design by evolutionary mechanisms found in biological species, simulated annealing is based on the analogy of the cooling and annealing of metals. Both genetic algorithm and simulated annealing have been used for solving numerous problems from a wide range of application domains and have been found to be very effective and robust throughout, and are even suitable for ill-posed problems where some of the parameters are not known before hand. In this paper we propose simulated annealing based probability conditions to generate a new population in genetic algorithm. The proposed algorithm does not make use of the mutation operation. The new algorithm is validated with standard test cases and the results are compared with genetic algorithm and simulated annealing algorithm. The results obtained indicate a promising performance of the new approach in genetic algorithm.
\end{abstract}

AMS subject classification:

Keywords: optimization; genetic algorithm; simulated annealing. 


\section{Introduction}

Genetic algorithm (GA) is a partial search algorithm inspired by observing the biological evolution processes such as reproduction, recombination and mutation. The application of genetic algorithm has been successful in generating sufficiently close approximate solutions to various optimization problems. The genetic algorithm is designed in such a way that a solution of the problem naturally evolves when the parameters of the problem are suitably tweaked. The applications of genetic algorithm are wide and diverse and include areas such as engineering, art, biology, economics, marketing, genetics, operations research, robotics, social sciences, physics, politics and chemistry. Genetic algorithms have been successfully used in numerical function optimization [1], combinatorial optimization [2], image processing [3], machine learning ([4, 5, 6]), data mining, information retrieval and grammar induction.

The building block theory of GA that was proposed by John Henry Holland [7] in the 1970s for solving practical optimization problems and are well documented ([8, 3, 9, 10, 11]). A basic genetic algorithm comprises of three genetic operators, namely, selection, crossover and mutation. GA aims to achieve quality results through selection, crossover and mutation. The effectiveness of GA can be improved by the correct design of the search operators and their appropriate integration to the GA. It aims the best solution which would be a global optimum and is achieved by maintaining the population of search operators fairly diverse. There is a lot of literature to improve the effectiveness of the GA operators (see $[8,3,12,13,14,15,16,17,18,19,20,18,21,1])$.

The overall GA process steps are summarized as follows:

STEP 1 Choose coding to represent problem parameters: a selection operator, a crossover operator and a mutation operator.

STEP 2 Choose initial population size, length of a string, probabilities of cross over and mutation, search domain of the variables, termination criteria or maximum number of iteration.

STEP 3 Set $T=0$. Generate initial population from the search domains randomly.

STEP 4 Evaluate each string of the population for fitness.

STEP 5 If termination criteria is satisfied, or $T>\operatorname{Tmax}$, then STOP.

STEP 6 Perform selection on the population.

STEP 7 Perform crossover on random pairs of strings.

STEP 8 Perform mutation on every string.

STEP 9 Evaluate the strings of the new population.

STEP 10 Set $T=T+1$, go to step 4 . 


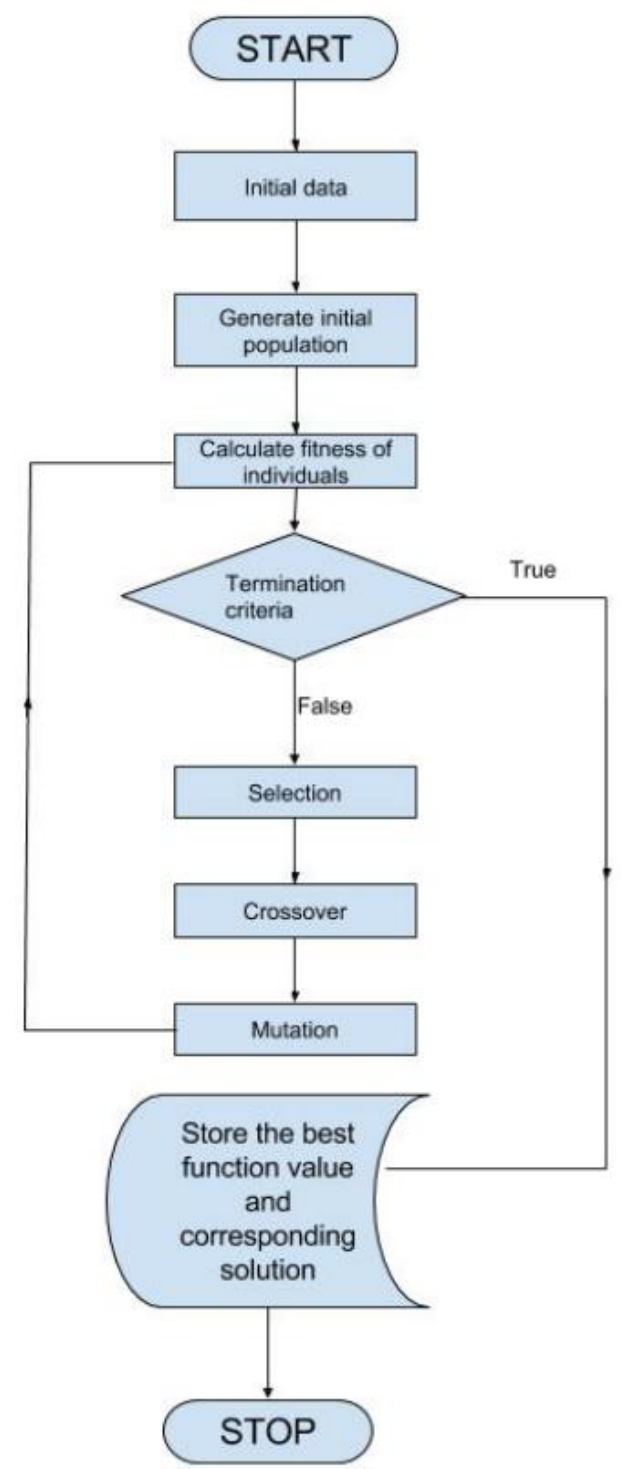

Figure 1: Flowchart for GA

Fig. 1 shows the schematic of the application of genetic algorithm.

Simulated annealing (SA) is a randomized perturbation technique, which often generates excellent solutions to various optimization problems. The main advantage of SA is the substantial reduction in computation time for most optimization problems. The basic strategy behind simulated annealing is to start from an arbitrarily selected solution and keep generating improved solutions by slightly perturbing it. Each time a solution is generated, a cost function evaluates its optimality, based on which it is accepted or 
allowed to move onto an improved solution according to an acceptance rule.

SA has received a lot of attention in the field of solving various optimization problems. Based on a theory proposed by Metropolis et al. [22], Kirkpatrick et al. [23] and Cerny [24] exhibited a model for simulating the annealing of solids. Many of the desciplines in Engineering such as integrated circuit placement, VLSI design, pattern recognition, optimal encoding, resource allocation, logic synthesis use SA solution. At the same time, theoretical studies have been focusing on the reasons for the excellent behaviour of the algorithm. There is an extensive literature on the strategy and its convergent properties, which can be found in $[25,26,27,28,29,30,31,32,33,34,35,36,37,38,39,40,41,42]$.

The overall SA process steps are summarized as follows:

STEP 1 Select an initial temperature $T$.

STEP 2 Select a randomly generated initial solution vector, $X$, and generate the objective function.

STEP 3 Select the temperature change counter.

STEP 4 Select a temperature cooling schedule.

STEP 5 Select a repetition schedule that defines the number of iterations executed at each temperature.

STEP 6 Give a random perturbation on $X$ and generate a new solution vector, $Y$, in the neighbourhood of current solution vector, $X$, evaluate the objective function.

STEP 7 If $f(Y)<f(X)$, replace $X$ with $Y$. Update the existing optimal solution and go to Step 9.

STEP 8 Else accept $Y$ with the probability $p=e^{-\frac{\Delta f}{T}}$ where $\Delta f=f(Y)-f(X)$. If the solution is accepted, replace $X$ with $Y$. Update the existing optimal solution and go to Step 9.

STEP 9 Decrease the temperature periodically.

STEP 10 Repeat Steps $6-9$ until stopping criterion is met.

In this paper, we propose a simulated annealing probability condition based new selection operator for accepting the offspring in genetic algorithm; and an integrated algorithm which integrates the new selection operator with other GA operators. Further, the integrated GA, which we call GASA, is free of mutation operator, and which helps to reduce the number of iterations for convergence substatially. Thereafter, in section III, the computational results of test problems are shown. Finally, section IV summarizes the concluding remarks. 


\section{GASA algorithm}

In the proposed GASA algorithm, we generate the initial population randomly and create mating pool using selection operator. A pair (called parents) is chosen randomly from the selected mating pool for the crossover operation from which two offsprings are created. An offspring is accepted in the new population if the function value of the offstring has a better value than one of its parents or probability conditions used in simulated annealing algorithm is satisfied. The process of creating offsprings terminates when the desired number of individuals in the new population is satisfied. In each generation, the new population consists of offsprings produced from mating individuals from the current population have better quality either by functionally or probabilistically.

The step by step procedure of GASA can be summarized as follows:

STEP 1 Choose coding to represent problem parameters: a selection operator and a crossover operator

STEP 2 Choose initial population size, length of a string, probability for cross over , search domain of the variables, an initial temperature $T$, the temperature change counter, temperature cooling schedule, termination criteria.

STEP 3 Generate initial population from the search domains randomly.

STEP 4 Evaluate each string of the population for fitness.

STEP 5 If termination criteria is satisfied, THEN jump to STEP-12. ELSE Jump to STEP 6.

STEP 6 Perform selection on the population to create mating pool.

STEP 7 Randomly choose a pair from the selected mating pool, say $A$ and $B$. Perform crossover on $A$ and $B$ to produce two children, say, $X$ and $Y$.

STEP 8 Accept $X$ if $f(X)<f(A)$ or $f(X)<f(B)$ or, accept $X$ with the probability $p=e^{-\frac{\Delta f}{T}}$ where $\Delta f=f(X)-f(A)$ or $\Delta f=f(X)-f(B)$

STEP 9 Accept $Y$ if $f(Y)<f(A)$ or $f(Y)<f(B)$ or, accept $Y$ with the probability $p=e^{-\frac{\Delta f}{T}}$ where $\Delta f=f(Y)-f(A)$ or $\Delta f=f(Y)-f(B)$

STEP 10 Repeat the steps 7 to 9 until a new population with desired population size is obtained.

STEP 11 Decrease the temperature periodically. Go to STEP 4

STEP 12 Store best value and corresponding solution. Then STOP.

The flowchart for GASA is shown in Fig. 2. 


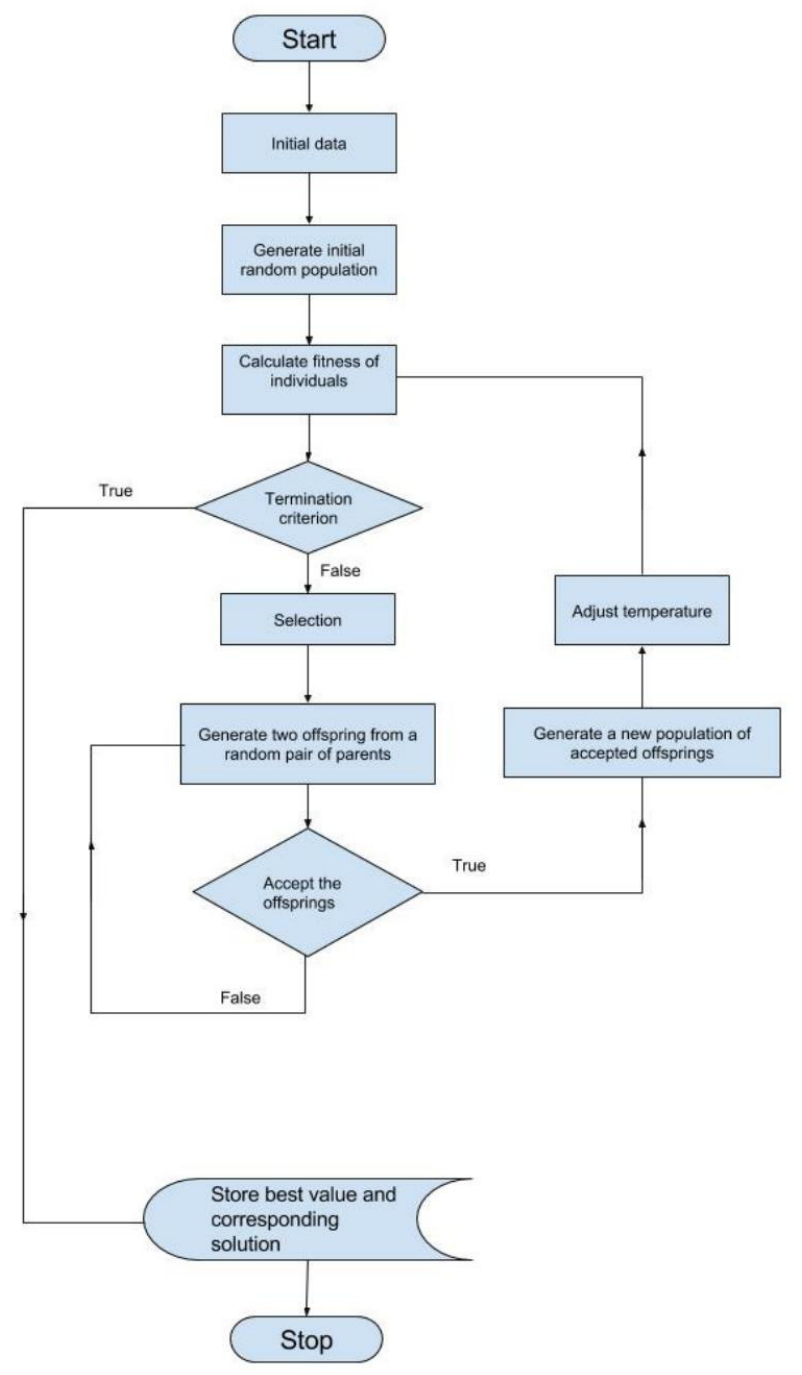

Figure 2: Flowchart for GASA

\section{Case studies}

We evaluate the performance of the proposed GASA algorithm, standard GA and SA on a set of 15 benchmark test functions (see Table 1 and Table 2) in the literature with different complexity. Experiments are conducted on the $2-D$ problems. The results of GASA, GA and SA are compared. Parameters used in the implementation of GASA, GA and SA are : Population Size $=60$, bit length for one variable $=16$, probability of crossover $=0.80$, maximum number of generations for convergence $=100$, alternative tolerance for convergence $=0.0000000001$, the initial temperature $=1$, temperature at which to stop $=e^{-8}$, maximum number of tries within one temperature $=3$.

We use the MATLAB to implement the algorithms. All the implementations were 

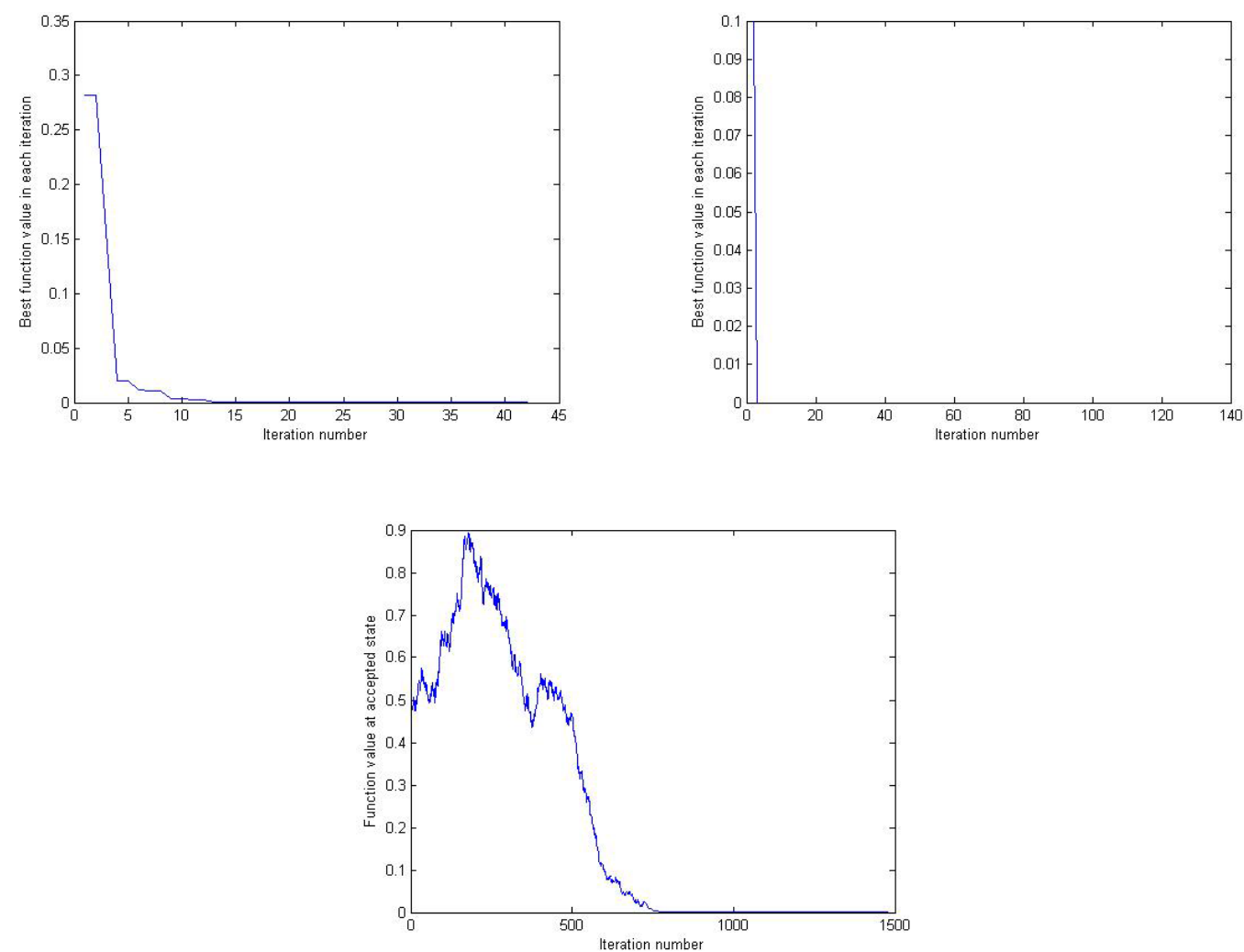

Figure 3: Convergence Graph for the function $g_{1}$ using GASA, GA and SA algorithms. Left most plot is for GASA algorithm, middle plot is for GA algorithm and right most plot is for SA algorithm.

developed using a computer with processor Core 2 Quad processor of 64 bits that works to a frequency of $2.7 \mathrm{GHz}, 4 \mathrm{~GB}$ of RAM Memory and Windows XP Professional Version.

In table 3, we can find the experimental results. The table 3 provides the results with 50 Monte-Carlo simulations with the changes in the random sequence. The results are provided in terms of statistical parameters such as average, the median, the best value, the worst value and standard deviation obtained for each test functions. The number of generations for convergence of the functions taken by the two methods GASA and GA is shown in table 4. The convergence maps of the algorithm on GASA, GA and SA functions $g_{1}$ to $g_{15}$ are plotted in figures 3-17 respectively.

The experimental analysis shows that the GASA algorithm converges quickly to the optimal solution and thus exhibits a superior performance in comparison to GA and SA algorithms. 
Table 1: Test functions $g_{1}$ to $g_{12}$

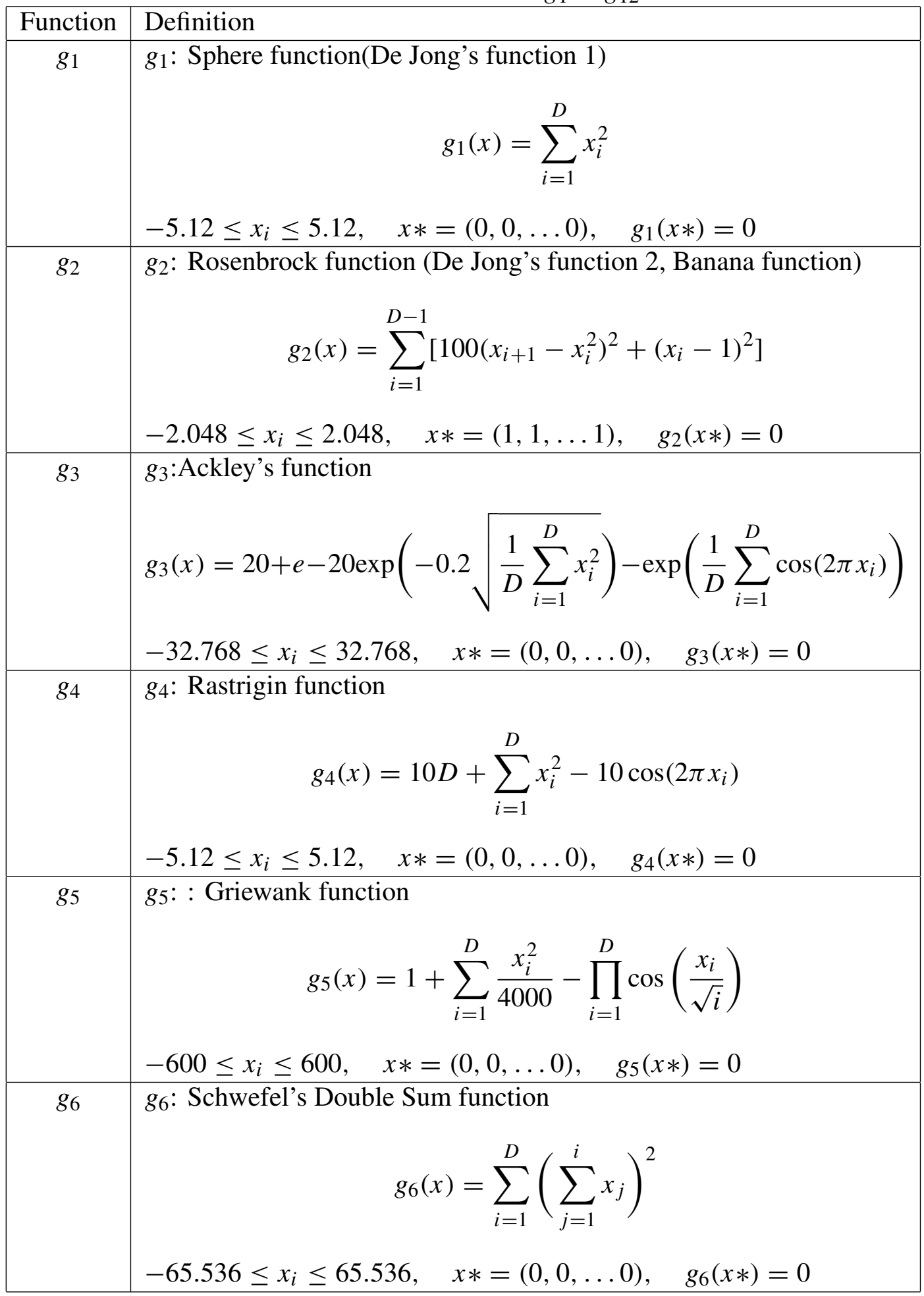


Table 1: Continued.

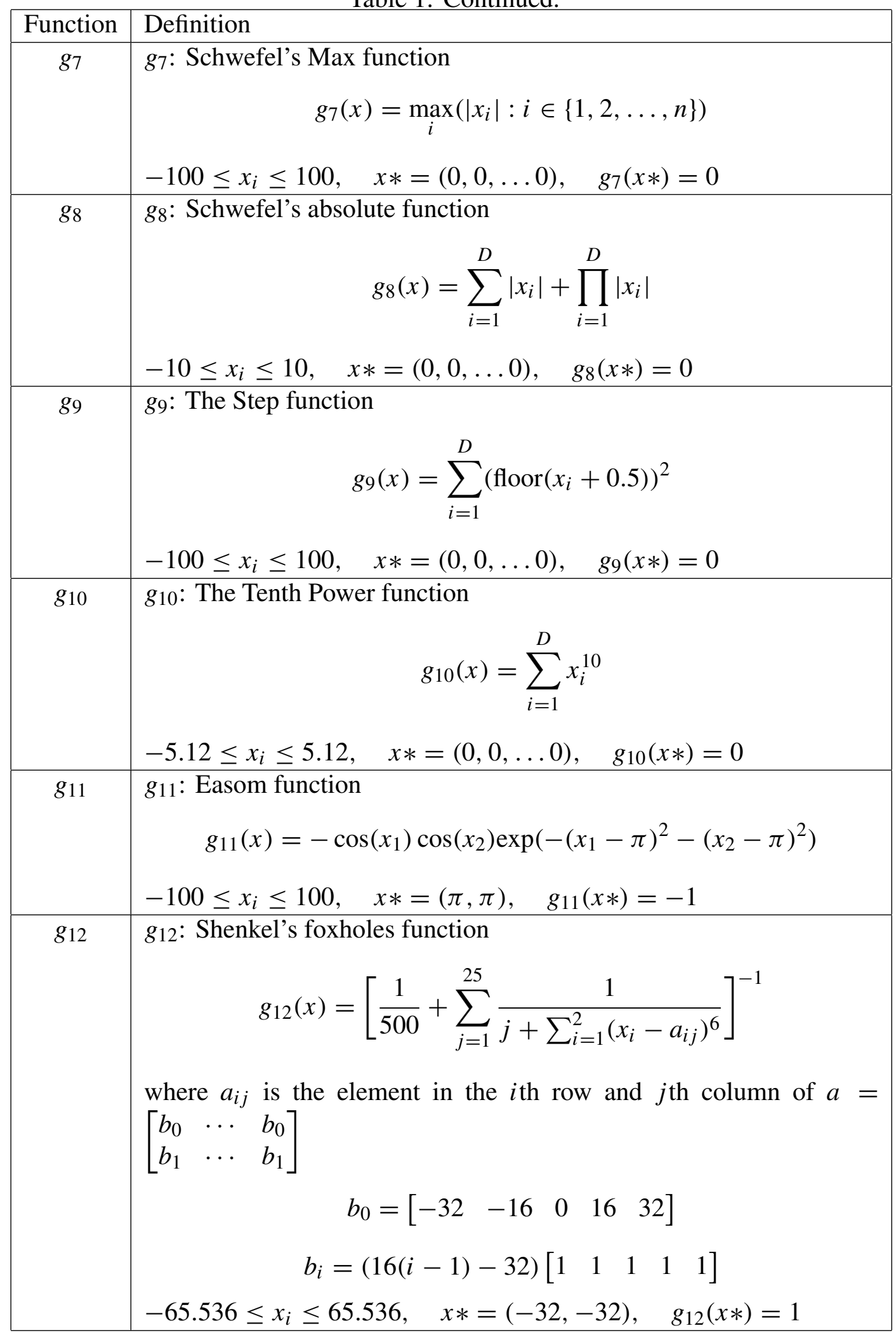


Table 2: Test functions $g_{13}$ to $g_{15}$

\begin{tabular}{|c|c|}
\hline Function & Definition \\
\hline \multirow[t]{4}{*}{$g_{13}$} & $g_{13}:$ Goldstein-Price function \\
\hline & $g_{13}(x)=\left[1+\left(x_{1}+x_{2}+1\right)^{2}\left(19-14 x_{1}+3 x_{1}^{2}-14 x_{2}+6 x_{1} x_{2}+3 x_{2}^{2}\right)\right]$ \\
\hline & $\times\left[30+\left(2 x_{1}-3 x_{2}\right)^{2}\left(18-32 x_{1}+12 x_{1}^{2}+48 x_{2}-36 x_{1} x_{2}+27 x_{2}^{2}\right)\right]$ \\
\hline & $-2 \leq x_{i} \leq 2, \quad x *=(0,-1), \quad g_{13}(x *)=3$ \\
\hline \multirow[t]{3}{*}{$g_{14}$} & $g_{14}:$ Six hump camel back function \\
\hline & $g_{14}(x)=\left[\left(4-2.1 x_{1}^{2}+x_{1}^{4 / 3}\right) x_{1}^{2}+x_{1} x_{2}+\left(-4+4 x_{2}^{2}\right) x_{2}^{2}\right.$ \\
\hline & 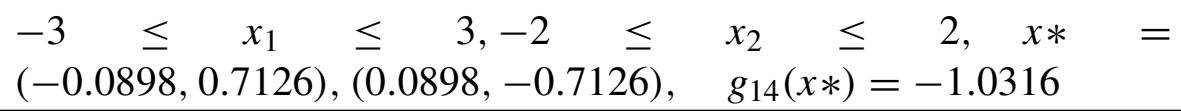 \\
\hline \multirow[t]{3}{*}{$g_{15}$} & $g_{15}:$ Branin's rcos function \\
\hline & $g_{15}(x)=\left(x_{2}-\frac{5.1}{4 \pi^{2}} x_{1}^{2}+\frac{5}{\pi} x_{1}-6\right)^{2}+\left(10-\frac{5}{4 \pi}\right) \cos x_{1}+10$ \\
\hline & 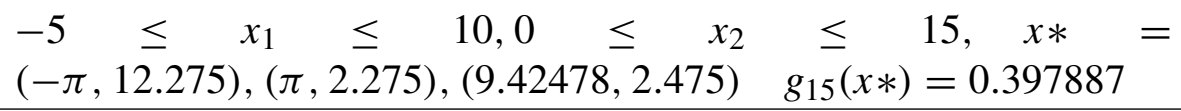 \\
\hline
\end{tabular}

Table 3: Comparison results of the best value, the median, the worst value, average, and standard deviation of the functions $g_{1}-g_{15}$ for 50 independent runs using GASA, GA and SA methods

\begin{tabular}{|c|c|c|c|c|c|c|}
\hline F & Method & Best & Median & Worse & Average & S.D \\
\hline \multirow{3}{*}{$g_{1}$} & GASA & $1.1642 \mathrm{E}-10$ & $1.1642 \mathrm{E}-08$ & $1.1823 \mathrm{E}-08$ & $1.1435 \mathrm{E}-08$ & $1.6885 \mathrm{E}-24$ \\
\cline { 2 - 8 } & GA & $4.6568 \mathrm{E}-06$ & $4.6568 \mathrm{E}-06$ & $2.3284 \mathrm{E}-05$ & $1.3970 \mathrm{E}-05$ & $9.4081 \mathrm{E}-06$ \\
\cline { 2 - 8 } & SA & $1.4136 \mathrm{E}-10$ & $1.1834 \mathrm{E}-08$ & $4.9742 \mathrm{E}-08$ & $1.2281 \mathrm{E}-09$ & $7.1842 \mathrm{E}-06$ \\
\hline \multirow{4}{*}{$g_{2}$} & GASA & $2.3516 \mathrm{E}-08$ & $2.9087 \mathrm{E}-08$ & $5.1371 \mathrm{E}-08$ & $3.3098 \mathrm{E}-08$ & $9.5848 \mathrm{E}-09$ \\
\cline { 2 - 8 } & GA & $7.2552 \mathrm{E}-07$ & $1.8174 \mathrm{E}-05$ & $5.1102 \mathrm{E}-05$ & $2.2489 \mathrm{E}-05$ & $1.5667 \mathrm{E}-05$ \\
\cline { 2 - 8 }$g_{3}$ & SA & $1.8498 \mathrm{E}-06$ & $8.1437 \mathrm{E}-06$ & $5.4235 \mathrm{E}-05$ & $9.8367 \mathrm{E}-06$ & $4.0757 \mathrm{E}-05$ \\
\hline \multirow{3}{*}{$g_{4}$} & GASA & 0.0020 & 0.0020 & 0.0020 & 0.0020 & $3.4156 \mathrm{e}-35$ \\
\cline { 2 - 8 } & GA & 0.0020 & 0.0020 & 0.0034 & 0.0023 & $8.6401 \mathrm{E}-04$ \\
\cline { 2 - 8 } & SA & $9.7133 \mathrm{E}-07$ & $4.4241 \mathrm{E}-05$ & $7.4176 \mathrm{E}-04$ & $1.7511 \mathrm{E}-05$ & $3.2534 \mathrm{E}-05$ \\
\cline { 2 - 8 } & GASA & $2.3097 \mathrm{E}-08$ & $2.3097 \mathrm{E}-08$ & $1.6523 \mathrm{E}-06$ & $2.3549 \mathrm{E}-08$ & $7.2236 \mathrm{E}-24$ \\
\cline { 2 - 7 } & GA & $2.4219 \mathrm{E}-06$ & $1.2109 \mathrm{E}-05$ & $2.1797 \mathrm{E}-05$ & $1.1722 \mathrm{E}-05$ & $7.0457 \mathrm{E}-06$ \\
\cline { 2 - 7 } & SA & $2.6316 \mathrm{E}-08$ & $1.6317 \mathrm{E}-07$ & $1.6523 \mathrm{E}-06$ & $4.6412 \mathrm{E}-07$ & $1.8557 \mathrm{E}-05$ \\
\hline
\end{tabular}


Table 3: Continued.

\begin{tabular}{|c|c|c|c|c|c|c|}
\hline $\mathrm{F}$ & Method & Best & Median & Worse & Average & S.D \\
\hline \multirow{3}{*}{$g_{5}$} & GASA & $6.2907 \mathrm{E}-05$ & $6.2907 \mathrm{E}-05$ & $6.2907 \mathrm{E}-05$ & $6.2907 \mathrm{E}-05$ & $6.3582 \mathrm{E}-32$ \\
\hline & GA & 0.0061 & 0.0127 & 0.0143 & 0.0121 & 0.0023 \\
\hline & SA & 0.2929 & 0.2929 & 0.2929 & 0.2929 & $1.0121 \mathrm{E}-17$ \\
\hline \multirow{3}{*}{$g_{6}$} & GASA & 2.8374E-09 & $9.4386 \mathrm{E}-09$ & 9.8374E-08 & $2.9025 \mathrm{E}-09$ & $1.8174 \mathrm{E}-22$ \\
\hline & GA & $1.0000 \mathrm{E}-06$ & $1.3000 \mathrm{E}-05$ & $2.5001 \mathrm{E}-05$ & $1.4360 \mathrm{E}-05$ & 7.0735E-06 \\
\hline & SA & $3.5179 \mathrm{E}-09$ & $6.1342 \mathrm{E}-09$ & $2.9201 \mathrm{E}-07$ & $9.3266 \mathrm{E}-08$ & $1.1271 \mathrm{E}-05$ \\
\hline \multirow{3}{*}{$g_{7}$} & GASA & 0.0015 & 0.0015 & 0.0015 & 0.0015 & $5.2345 \mathrm{E}-45$ \\
\hline & GA & 0.0015 & 0.0046 & 0.0076 & 0.0052 & 0.0022 \\
\hline & SA & $3.2158 \mathrm{E}-06$ & $5.1157 \mathrm{E}-05$ & $4.9007 \mathrm{E}-05$ & $1.0211 \mathrm{E}-05$ & $1.0193 \mathrm{E}-05$ \\
\hline \multirow{3}{*}{$g_{8}$} & GASA & $3.0520 \mathrm{E}-04$ & $3.0520 \mathrm{E}-04$ & $3.0520 \mathrm{E}-04$ & $3.0520 \mathrm{E}-04$ & $1.6598 \mathrm{E}-19$ \\
\hline & GA & $3.0520 \mathrm{E}-04$ & $9.1566 \mathrm{E}-04$ & $9.1575 \mathrm{E}-04$ & 7.5087E-04 & $2.4052 \mathrm{E}-04$ \\
\hline & SA & $1.9585 \mathrm{E}-07$ & $2.6243 \mathrm{E}-06$ & $6.1208 \mathrm{E}-06$ & $3.1147 \mathrm{E}-06$ & $3.1206 \mathrm{E}-05$ \\
\hline \multirow{3}{*}{$g_{9}$} & GASA & 0 & 0 & 0 & 0 & 0 \\
\hline & GA & 0 & 0 & 0 & 0 & 0 \\
\hline & SA & 0 & 0 & 0 & 0 & 0 \\
\hline \multirow{3}{*}{$g_{10}$} & GASA & $1.3053 \mathrm{E}-34$ & $4.0974 \mathrm{E}-29$ & $1.4874 \mathrm{E}-27$ & $3.4084 \mathrm{E}-28$ & 5.3894E-28 \\
\hline & GA & $5.2160 \mathrm{E}-29$ & $7.6683 \mathrm{E}-22$ & $2.2810 \mathrm{E}-20$ & $3.8371 \mathrm{E}-21$ & $5.8580 \mathrm{E}-21$ \\
\hline & SA & $3.6763 \mathrm{E}-14$ & $1.3712 \mathrm{E}-14$ & $7.6102 \mathrm{E}-13$ & $4.7126 \mathrm{e}-14$ & $3.1773 \mathrm{E}-05$ \\
\hline \multirow{3}{*}{$g_{11}$} & GASA & -1.0000 & -1.0000 & -1.0000 & -1.0000 & $2.4896 \mathrm{E}-05$ \\
\hline & GA & -1.0000 & -0.9995 & -0.9986 & -0.9995 & $3.5878 \mathrm{E}-04$ \\
\hline & SA & -1 & -1 & -0.9997 & -1 & 7.1214E-05 \\
\hline \multirow{3}{*}{$g_{12}$} & GASA & 0.9980 & 0.9980 & 0.9980 & 0.9980 & $3.3993 \mathrm{E}-16$ \\
\hline & GA & 0.9980 & 0.9980 & 0.9980 & 0.9980 & $1.9323 \mathrm{E}-10$ \\
\hline & SA & 0.9980 & 0.9980 & 0.9980 & 0.9980 & $1.2305 \mathrm{E}-16$ \\
\hline \multirow{3}{*}{$g_{13}$} & GASA & 3.0000 & 3.0000 & 3.0000 & 3.0000 & $1.1943 \mathrm{E}-48$ \\
\hline & GA & 3.0000 & 3.0000 & 3.0000 & 3.0000 & $1.2456 \mathrm{E}-05$ \\
\hline & SA & 3.0000 & 3.0000 & 3.0000 & 3.0000 & $1.2614 \mathrm{E}-05$ \\
\hline \multirow{3}{*}{$g_{14}$} & GASA & -1.0316 & -1.0316 & -1.0316 & -1.0316 & $2.2662 \mathrm{E}-16$ \\
\hline & GA & -1.0316 & -1.0316 & -1.0316 & -1.0316 & $1.3930 \mathrm{E}-05$ \\
\hline & SA & -1.0316 & -1.0316 & -1.0316 & -1.0316 & $1.2164 \mathrm{E}-08$ \\
\hline \multirow{3}{*}{$g_{15}$} & GASA & 0.3979 & 0.3979 & 0.3979 & 0.3979 & $1.1331 \mathrm{E}-16$ \\
\hline & GA & 0.3979 & 0.3979 & 0.3979 & 0.3979 & $1.9852 \mathrm{E}-06$ \\
\hline & $\mathrm{SA}$ & 0.397887 & 0.397887 & 0.397887 & 0.397887 & $1.1699 \mathrm{E}-06$ \\
\hline
\end{tabular}

F-Function 
Table 4: Comparison results - The number of generations for convergence of the functions $g_{1}-g_{15}$ using GASA and GA methods

\begin{tabular}{|c|c|c|c|c|c|c|c|c|c|c|c|c|c|c|c|c|}
\hline Function & & $g_{1}$ & $g_{2}$ & $g_{3}$ & $g_{4}$ & $g_{5}$ & $g_{6}$ & $g_{7}$ & $g_{8}$ & $g_{9}$ & $g_{10}$ & $g_{11}$ & $g_{12}$ & $g_{13}$ & $g_{14}$ & $g_{15}$ \\
\hline \multirow{3}{*}{ Method } & GASA & 42 & 92 & 60 & 54 & 68 & 80 & 35 & 70 & 5 & 8 & 17 & 86 & 62 & 40 & 72 \\
\cline { 2 - 36 } & GA & 136 & 172 & 62 & 85 & 110 & 89 & 49 & 142 & 5 & 10 & 19 & 99 & 114 \\
\hline
\end{tabular}
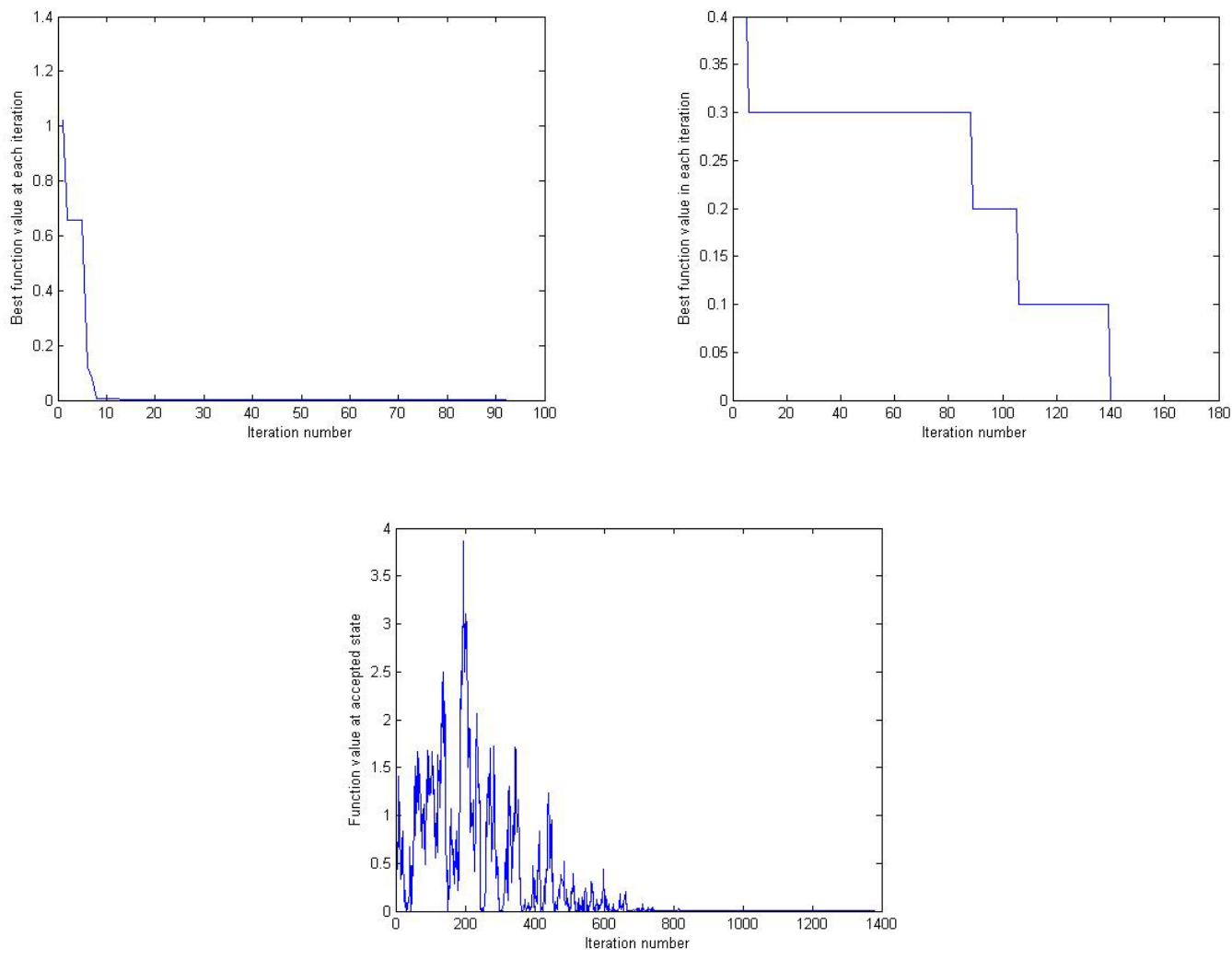

Figure 4: Convergence Graph for the function $g_{2}$ using GASA, GA and SA algorithms. Left most plot is for GASA algorithm, middle plot is for GA algorithm and right most plot is for SA algorithm. 

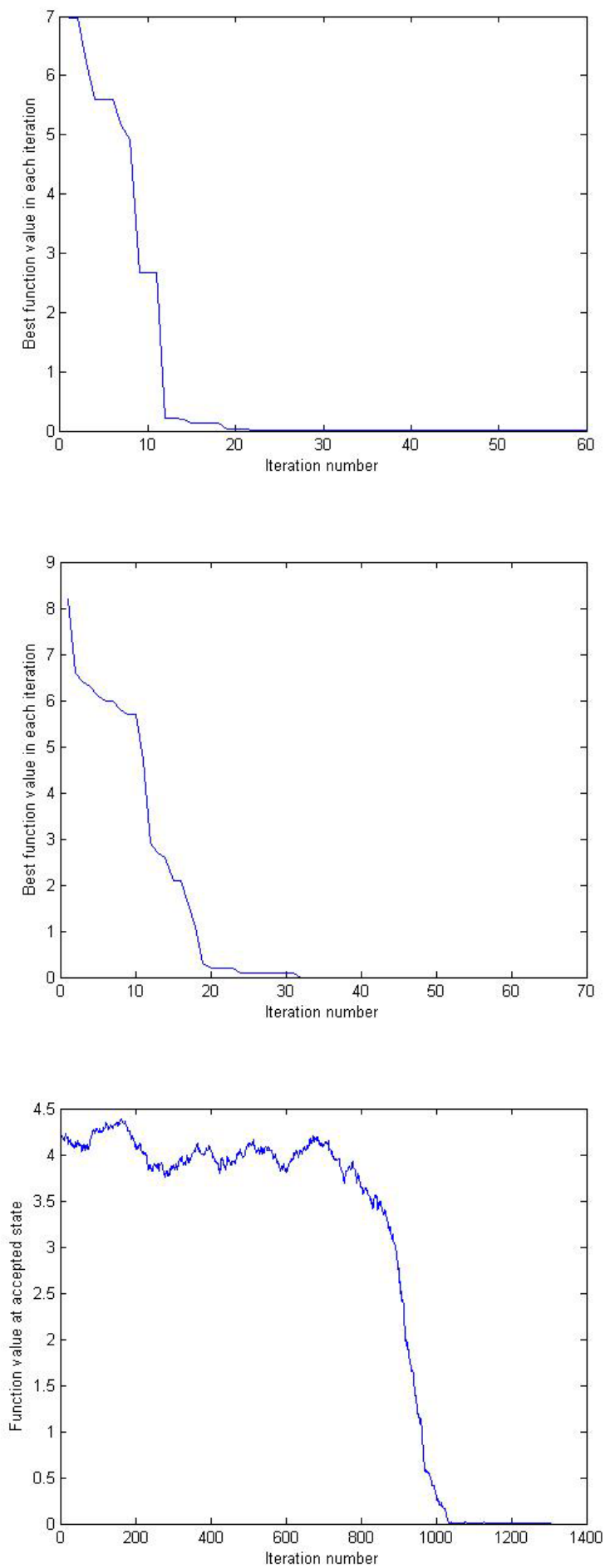

Figure 5: Convergence Graph for the function $g_{3}$ using GASA, GA and SA algorithms. Left most plot is for GASA algorithm, middle plot is for GA algorithm and right most plot is for SA algorithm. 

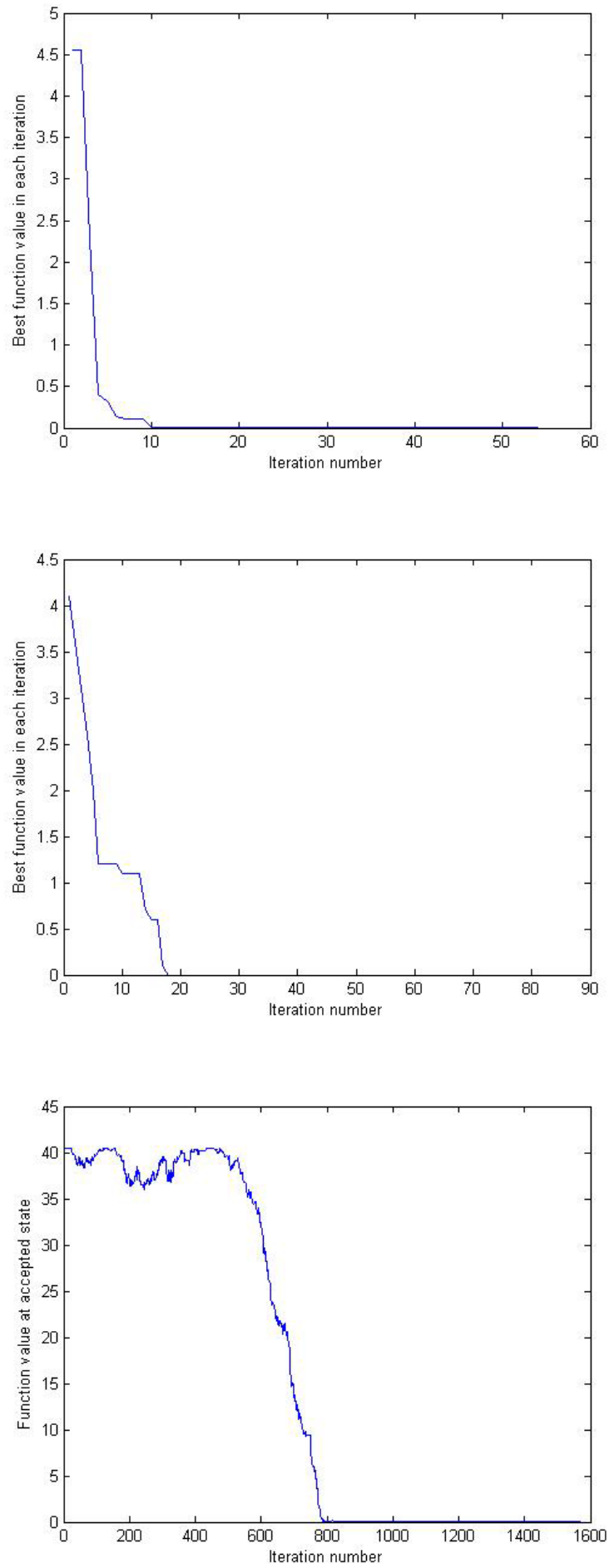

Figure 6: Convergence Graph for the function $g_{4}$ using GASA, GA and SA algorithms. Left most plot is for GASA algorithm, middle plot is for GA algorithm and right most plot is for SA algorithm. 

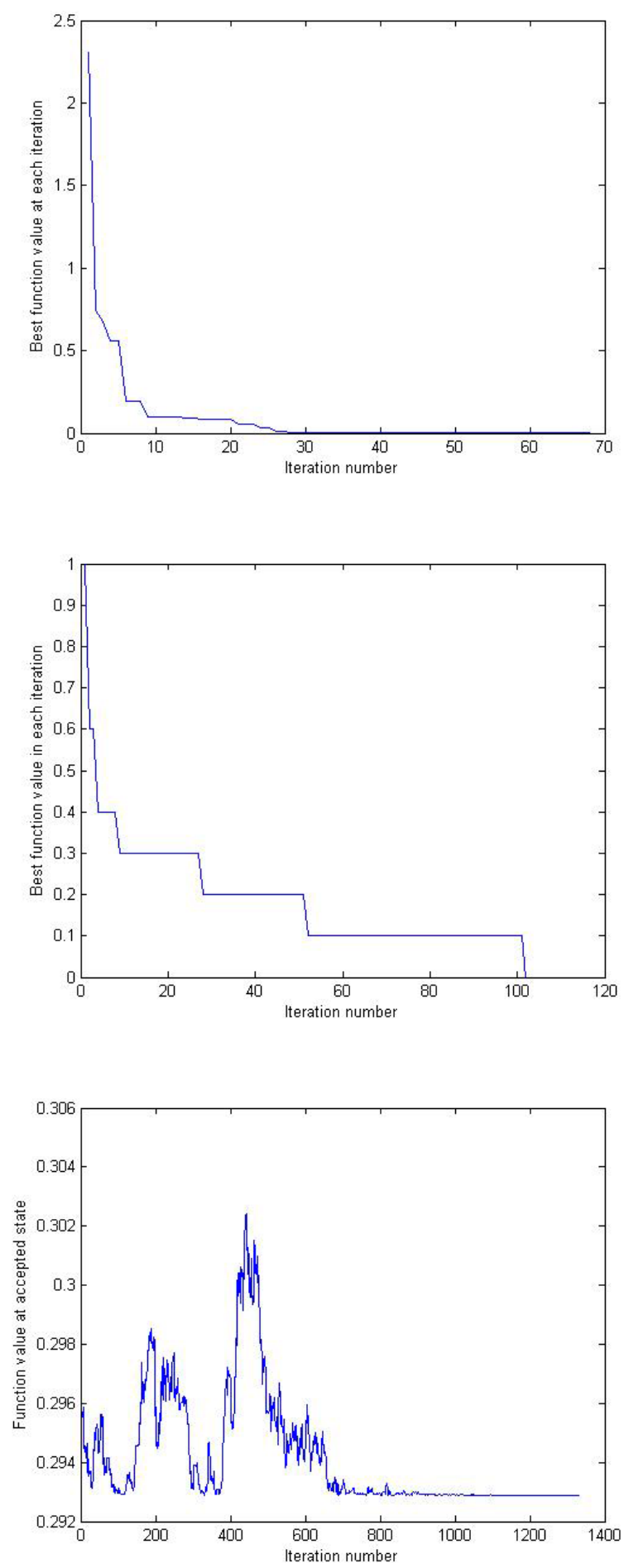

Figure 7: Convergence Graph for the function $g_{5}$ using GASA, GA and SA algorithms. Left most plot is for GASA algorithm, middle plot is for GA algorithm and right most plot is for SA algorithm. 

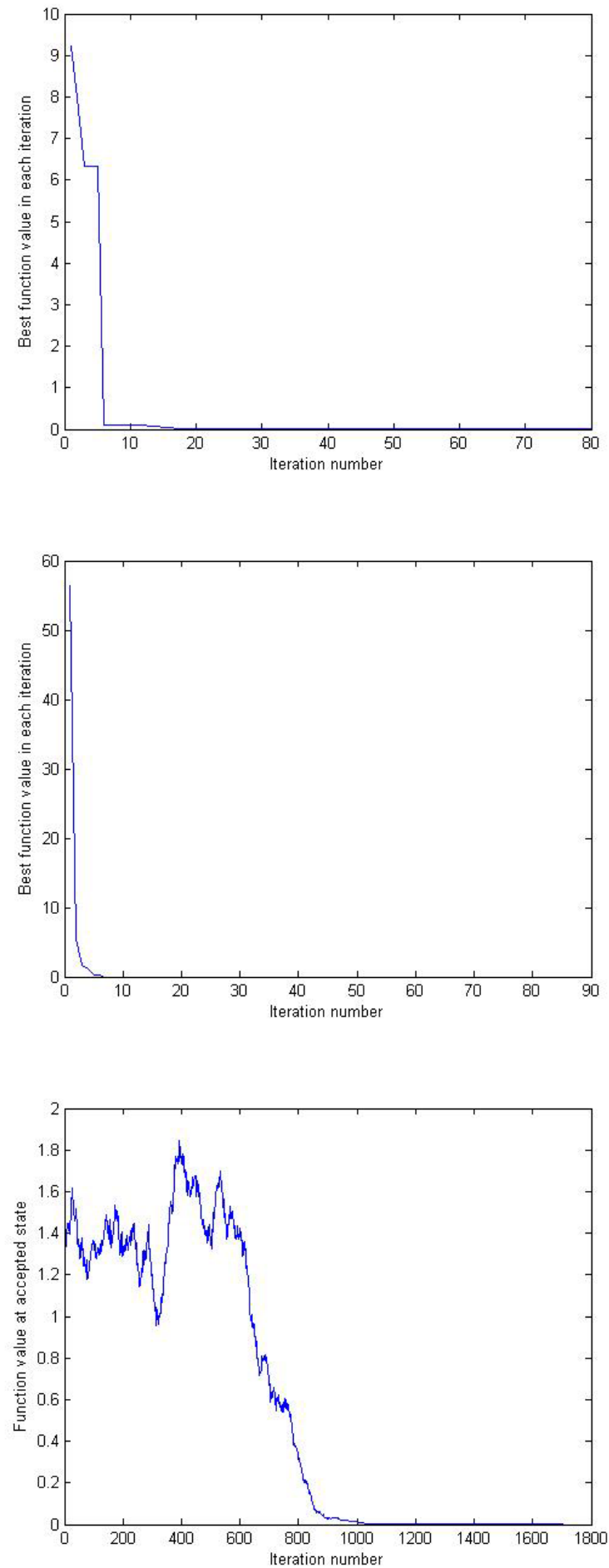

Figure 8: Convergence Graph for the function $g_{6}$ using GASA, GA and SA algorithms. Left most plot is for GASA algorithm, middle plot is for GA algorithm and right most plot is for SA algorithm. 

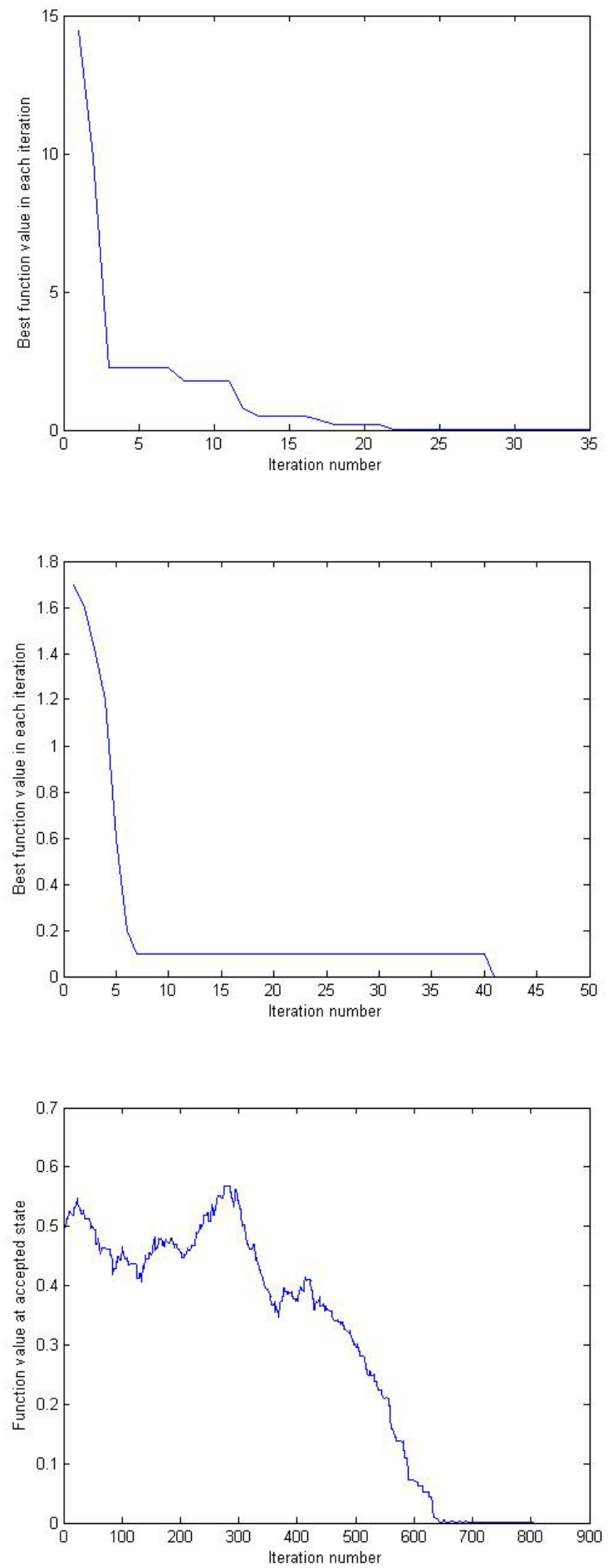

Figure 9: Convergence Graph for the function $g_{7}$ using GASA, GA and SA algorithms. Left most plot is for GASA algorithm, middle plot is for GA algorithm and right most plot is for SA algorithm. 

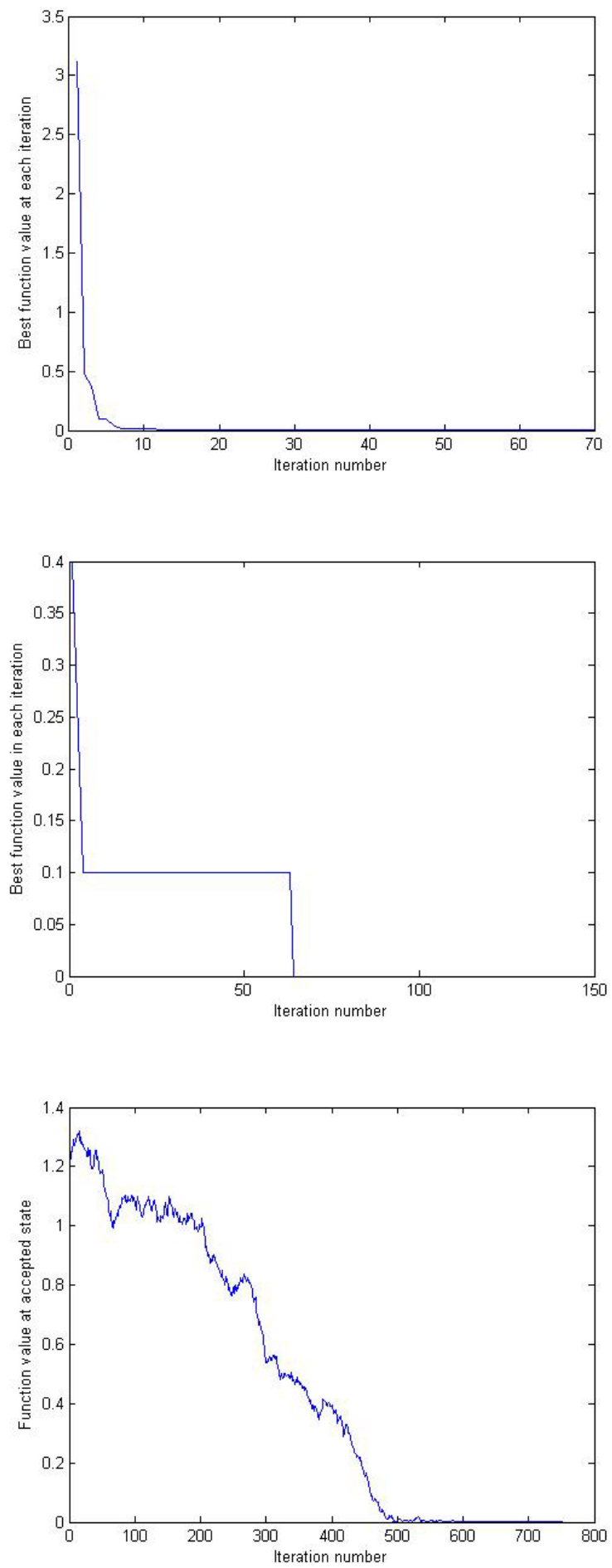

Figure 10: Convergence Graph for the function $g_{8}$ using GASA, GA and SA algorithms. Left most plot is for GASA algorithm, middle plot is for GA algorithm and right most plot is for SA algorithm. 

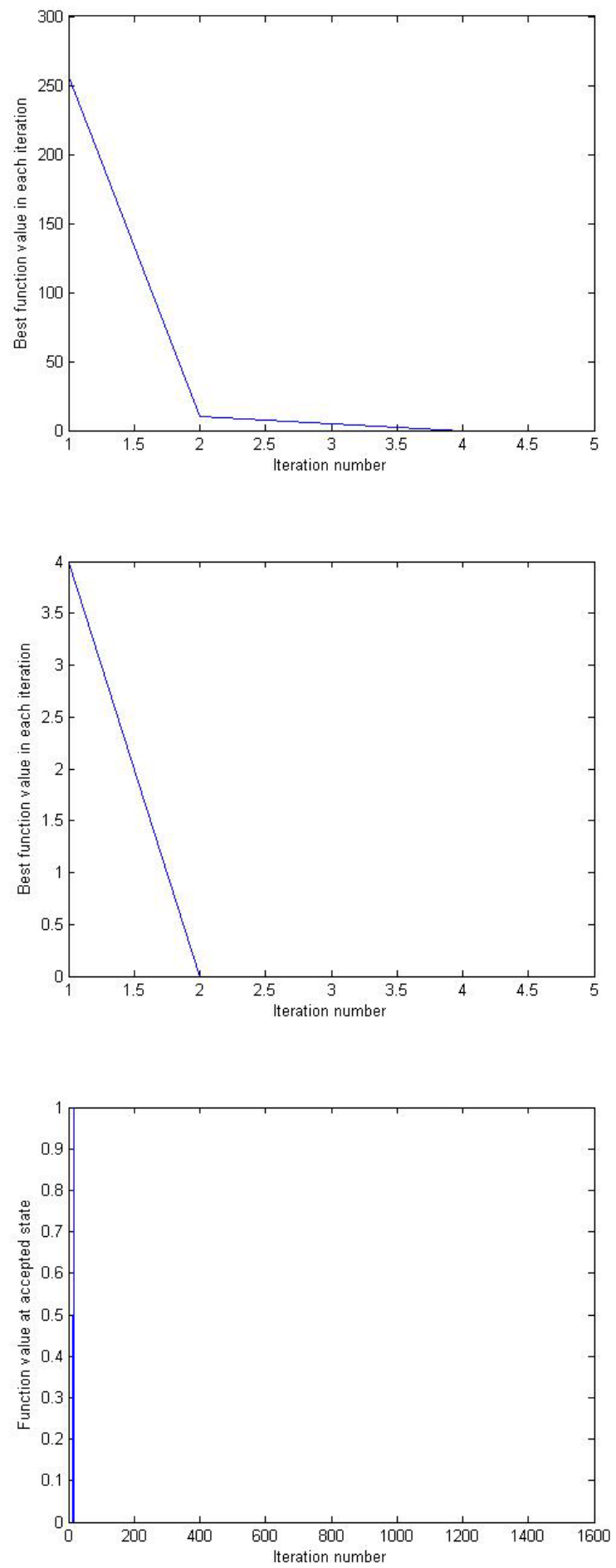

Figure 11: Convergence Graph for the function $g_{9}$ using GASA, GA and SA algorithms. Left most plot is for GASA algorithm, middle plot is for GA algorithm and right most plot is for SA algorithm. 

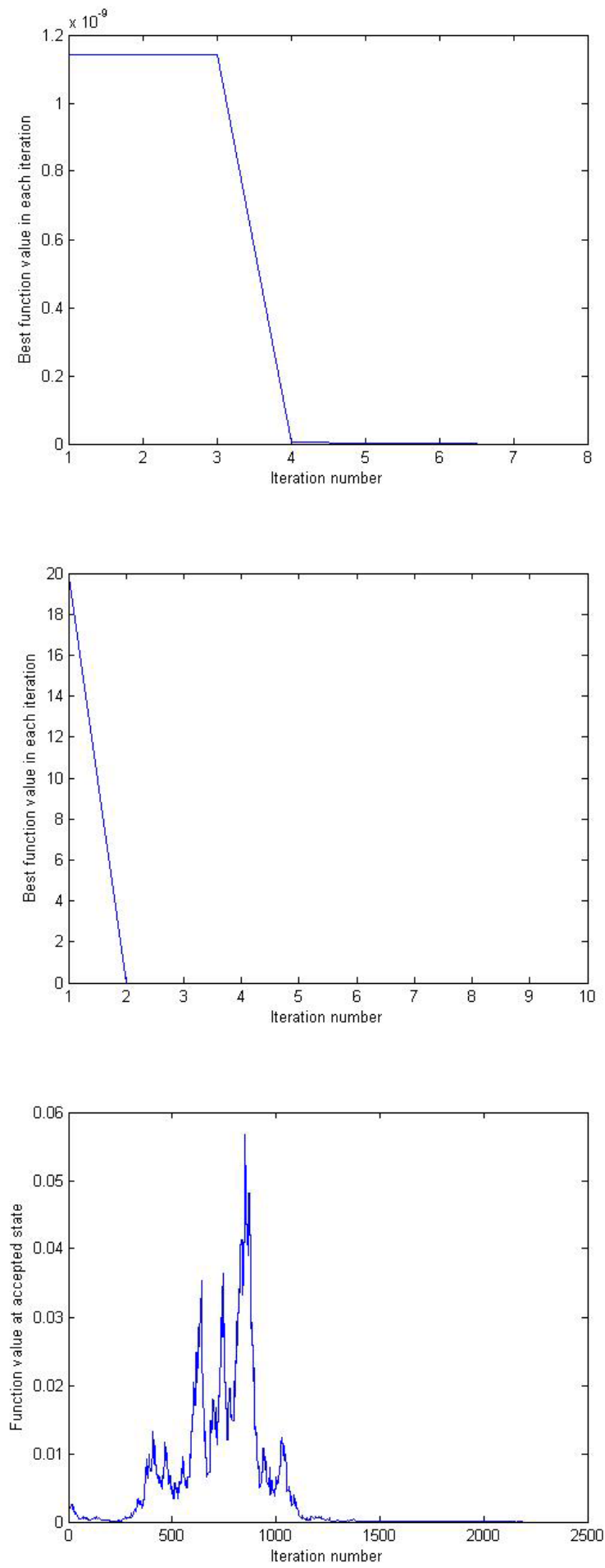

Figure 12: Convergence Graph for the function $g_{10}$ using GASA, GA and SA algorithms. Left most plot is for GASA algorithm, middle plot is for GA algorithm and right most plot is for SA algorithm. 

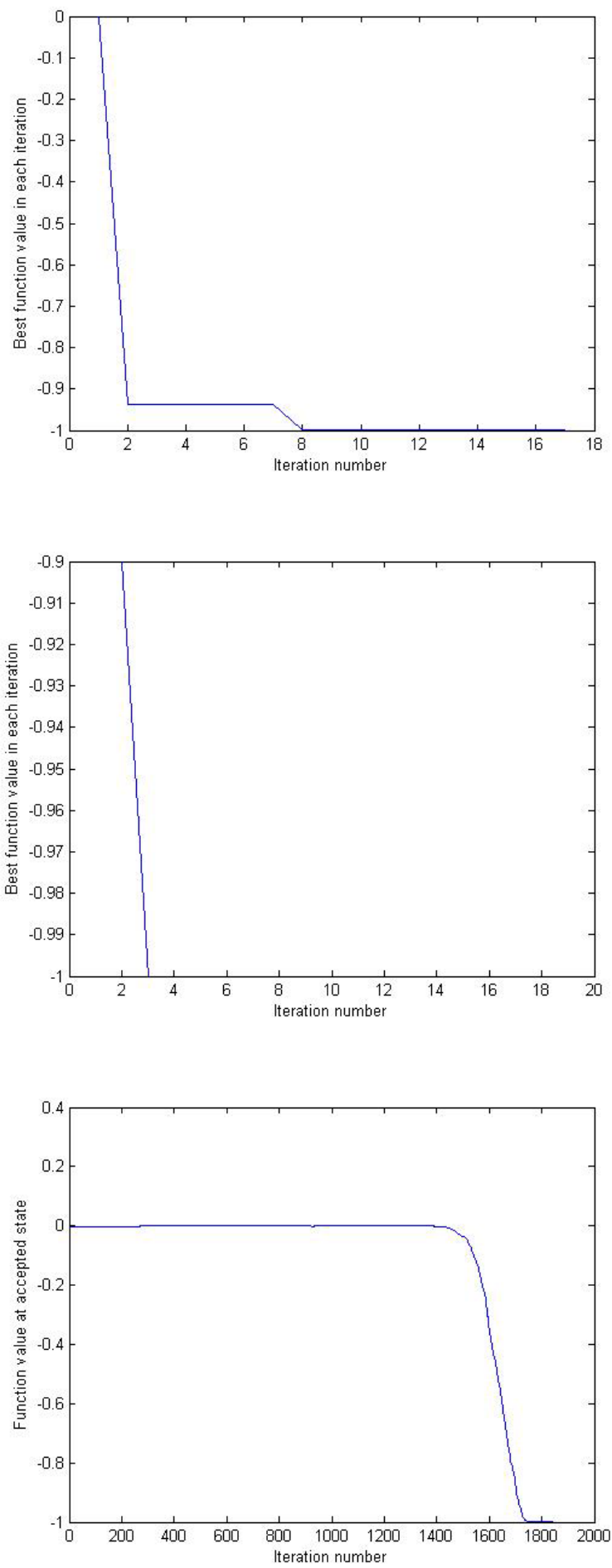

Figure 13: Convergence Graph for the function $g_{11}$ using GASA, GA and SA algorithms. Left most plot is for GASA algorithm, middle plot is for GA algorithm and right most plot is for SA algorithm. 

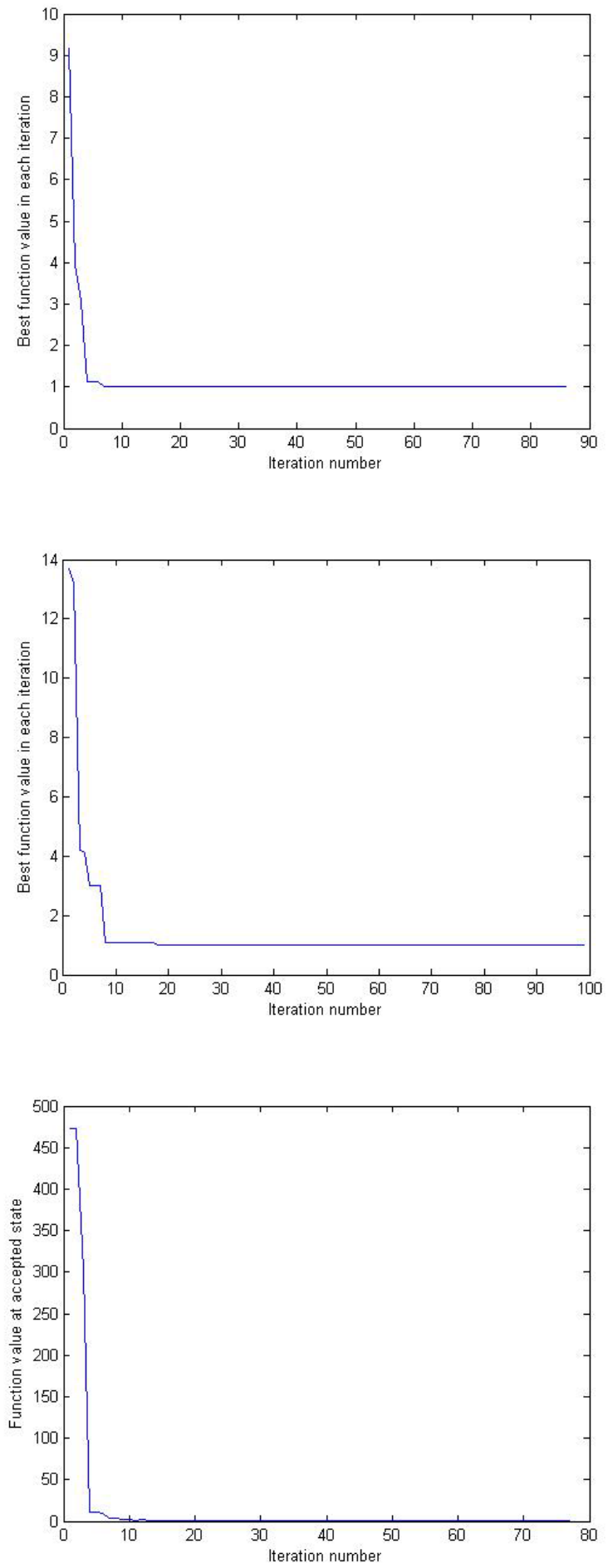

Figure 14: Convergence Graph for the function $g_{12}$ using GASA, GA and SA algorithms. Left most plot is for GASA algorithm, middle plot is for GA algorithm and right most plot is for SA algorithm. 

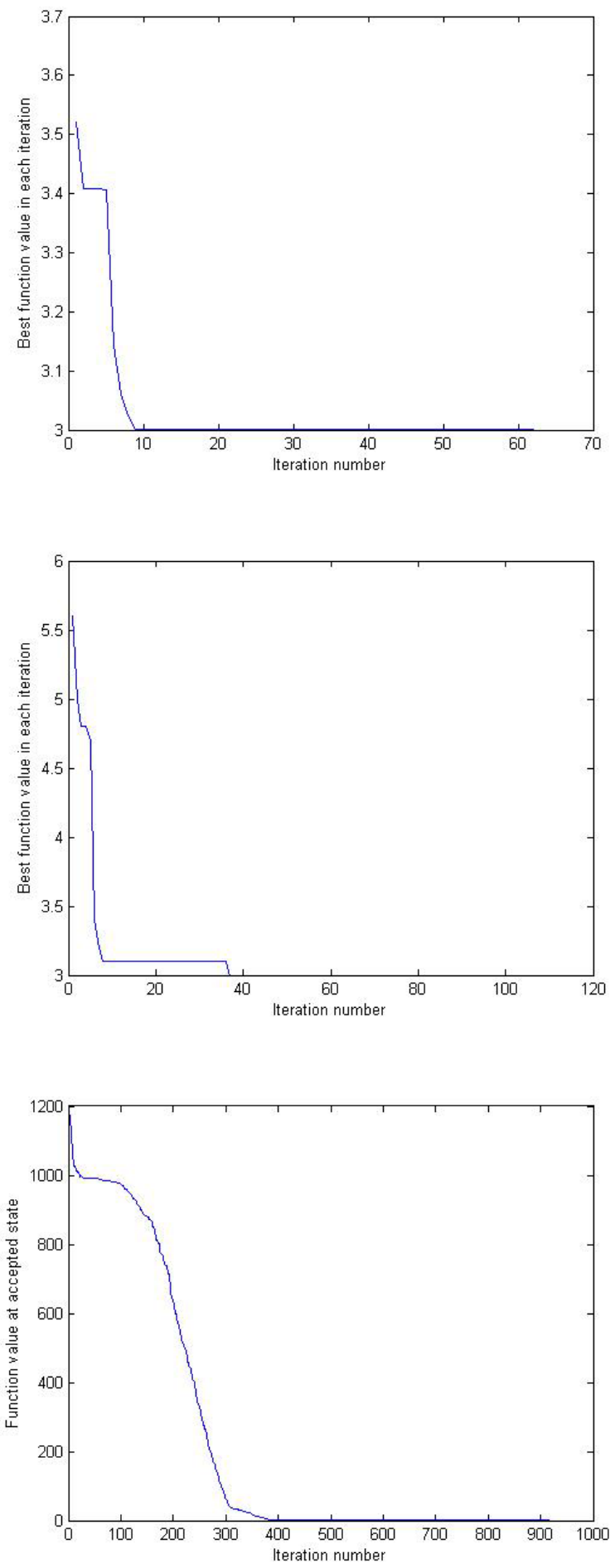

Figure 15: Convergence Graph for the function $g_{13}$ using GASA, GA and SA algorithms. Left most plot is for GASA algorithm, middle plot is for GA algorithm and right most plot is for SA algorithm. 

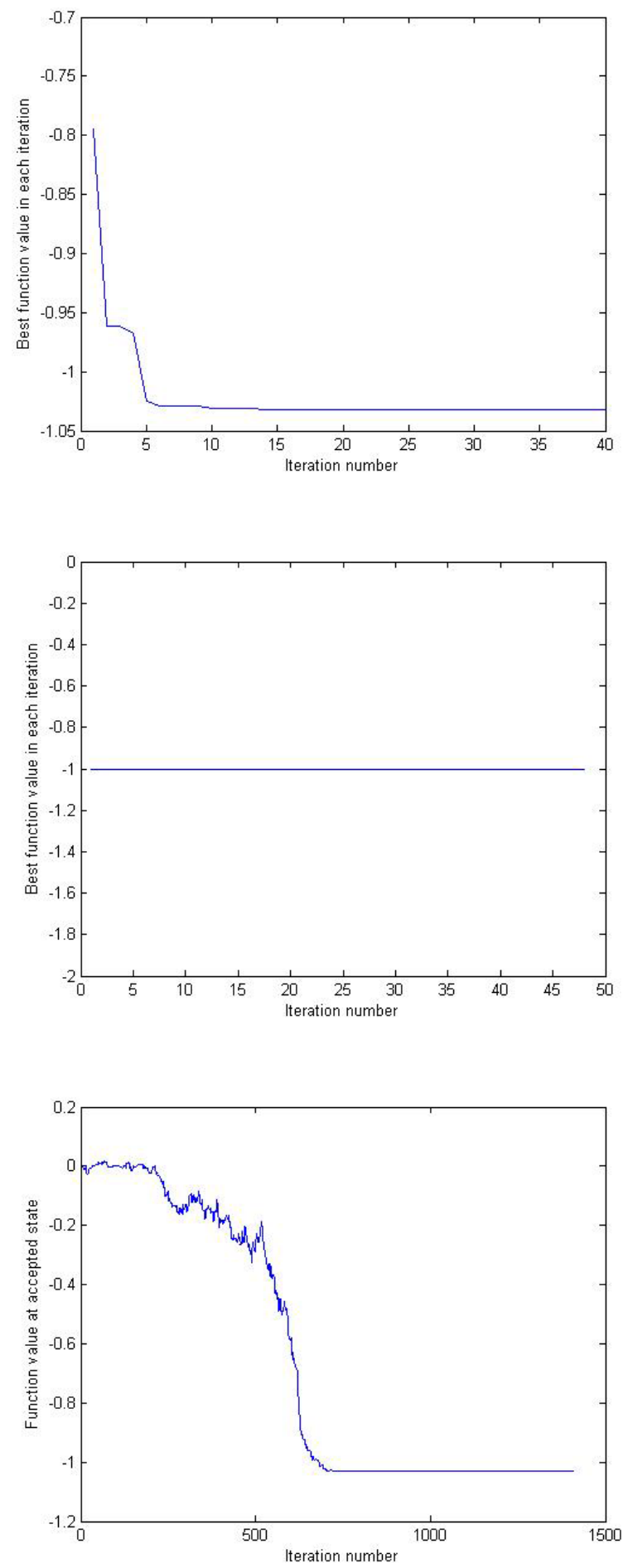

Figure 16: Convergence Graph for the function $g_{14}$ using GASA, GA and SA algorithms. Left most plot is for GASA algorithm, middle plot is for GA algorithm and right most plot is for SA algorithm. 

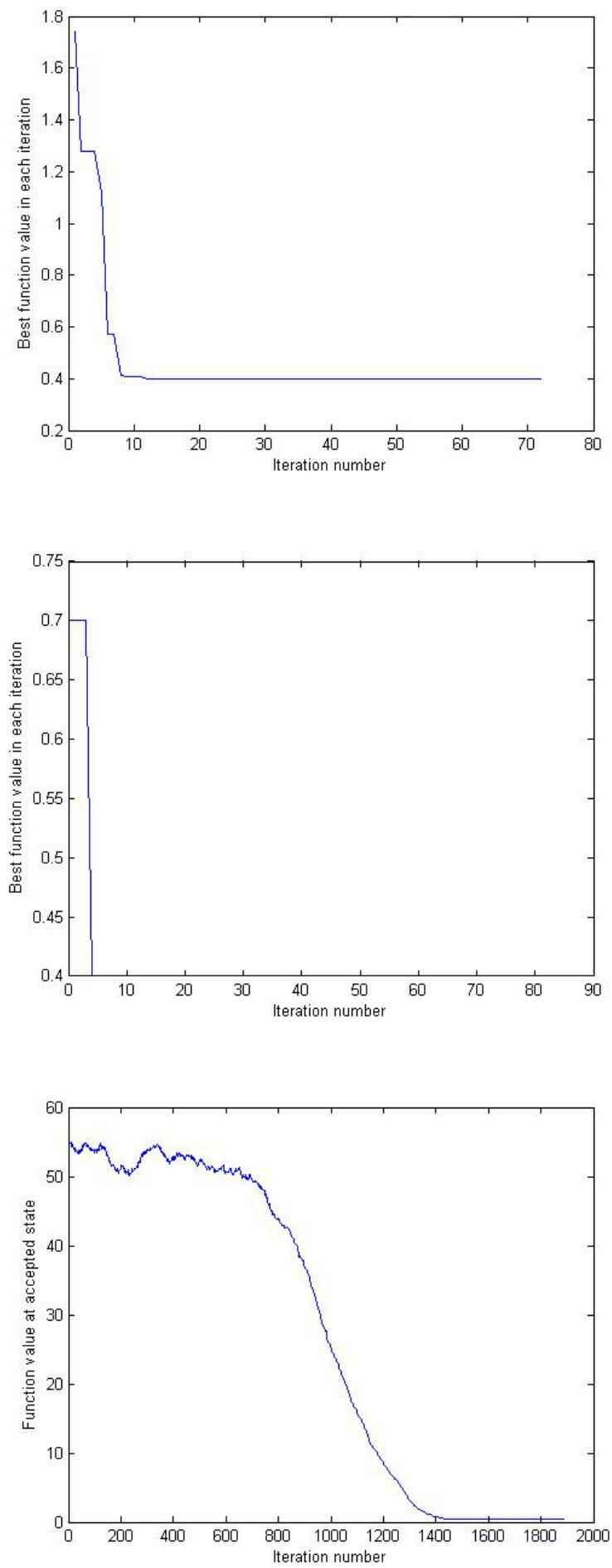

Figure 17: Convergence Graph for the function $g_{14}$ using GASA, GA and SA algorithms. Left most plot is for GASA algorithm, middle plot is for GA algorithm and right most plot is for SA algorithm. 


\section{Conclusion}

In this paper we have introduced simulated annealing based probability conditions to accept offsprings for creating a new population in genetic algorithm and have applied the integrated algorithm on a number of standard test cases. In the new approach the mutation operator is absent. We have simulated the results for the standard GA, SA and proposed GASA optimization method obtained by integrating the selection operator with the GA operators. The results of the three methods, namely proposed GASA method, GA method and SA, indicate that the former is capable of generating better solution quality than the other methods. It is seen that the GASA method significantly improve the convergence rate and the quality of the solution. In conclusion, it can be said that the proposed selection operator reduces the number of searches within the solution space and enhances the convergence capability and the performance of the GA.

\section{Acknowledgment}

The authors would like to thank Abhay Kumar, Group Director AFDG, S. Pandian, Deputy Director AERO and K. Sivan, Director VSSC for encouragement and support and also for providing the computational facilities.

\section{References}

[1] K. DeJong, The Analysis and behaviour of a Class of Genetic Adaptive Systems. Thesis (Ph.D.)-University of Michigan, 1975.

[2] D. Goldberg and R. Lingle, "Alleles, loci and the tsp en: Proceedings of the first international conference on genetic algorithms," 1985.

[3] D. Goldberg, Genetic Algorithms in Search, Optimization, and Machine Learning, ser. Artificial Intelligence. Addison-Wesley Publishing Company, 1989.

[4] K. D. Jong, "Adaptive system design: a genetic approach," Systems, Man and Cybernetics, IEEE Transactions on, vol. 10, no. 9, pp. 566-574, 1980.

[5] D. E. Goldberg and M. P. Samtani, "Engineering optimization via genetic algorithm," in Electronic Computation (1986). ASCE, 1986, pp. 471-482.

[6] D. E. Goldberg and C. H. Kuo, "Genetic algorithms in pipeline optimization," Journal of Computing in Civil Engineering, vol. 1, no. 2, pp. 128-141, 1987.

[7] J.H. Holland, Adaptation in natural and artificial systems: an introductory analysis with applications to biology, control, and artificial intelligence. U Michigan Press, 1975.

[8] L. Davis, "Genetic algorithms and simulated annealing," 1987.

[9] D. Beasley, R. Martin, and D. Bull, "An overview of genetic algorithms: Part 1. fundamentals," University computing, vol. 15, pp. 58-58, 1993.

[10] L. Davis, Handbook of genetic algorithms. New York: Van Nostrand Reinhold, 1991.

[11] T. Bäck, "Evolutionary algorithms in theory and practice," 1996. 
[12] J. E. Baker, "Adaptive selection methods for genetic algorithms," in Proceedings of an International Conference on Genetic Algorithms and their applications. Hillsdale, New Jersey, 1985, pp. 101-111.

[13] A. Brindle and U. of Alberta. Department of Computing Science, Genetic Algorithms for Function Optimization, ser. University of Alberta Department of Computing Science Technical Report Tr. Thesis (Ph.D.)-University of Alberta, 1981.

[14] D. Whitley, "Using reproductive evaluation to improve genetic search and heuristic discovery," in Genetic algorithms and their applications: proceedings of the second International Conference on Genetic Algorithms: July 28-31, 1987 at the Massachusetts Institute of Technology, Cambridge, MA. Hillsdale, NJ: L. Erlhaum Associates, 1987., 1987.

[15] J. E. Baker, "Reducing bias and inefficiency in the selection algorithm," in Proceedings of the second international conference on genetic algorithms, 1987, pp. $14-21$.

[16] L. D. Whitley et al., "The genitor algorithm and selection pressure: Why rank-based allocation of reproductive trials is best." in ICGA, vol. 89, 1989, pp. 116-123.

[17] G. Sywerda, "Uniform crossover in genetic algorithms," in Proceedings of the third international conference on Genetic algorithms. Morgan Kaufmann Publishers Inc., 1989, pp. 2-9.

[18] S. M. Elsayed, R. A. Sarker, and D. L. Essam, "A new genetic algorithm for solving optimization problems," Engineering Applications of Artificial Intelligence, vol. 27, pp. 57-69, 2014.

[19] D. E. Goldberg and K. Deb, "A comparative analysis of selection schemes used in genetic algorithms," Foundations of genetic algorithms, vol. 1, pp. 69-93, 1991.

[20] F. Liu and G. Zeng, "Study of genetic algorithm with reinforcement learning to solve the tsp," Expert Systems with Applications, vol. 36, no. 3, pp. 6995-7001, 2009.

[21] M. Thakur, S. S. Meghwani, and H. Jalota, "A modified real coded genetic algorithm for constrained optimization," Applied Mathematics and Computation, vol. 235, pp. 292-317, 2014.

[22] N. Metropolis, A. W. Rosenbluth, M. N. Rosenbluth, A. H. Teller, and E. Teller, "Equation of state calculations by fast computing machines," The journal of chemical physics, vol. 21, no. 6, pp. 1087-1092, 1953.

[23] S. Kirkpatrick, C. D. Gelatt, M. P. Vecchi et al., "Optimization by simulated annealing," Science, vol. 220, no. 4598, pp. 671-680, 1983.

[24] V. Černỳ, "Thermodynamical approach to the traveling salesman problem: An efficient simulation algorithm," Journal of optimization theory and applications, vol. 45, no. 1, pp. 41-51, 1985.

[25] D. Henderson, S. H. Jacobson, and A. W. Johnson, "The theory and practice of simulated annealing," in Handbook of metaheuristics. Springer, 2003, pp. $287-$ 319. 
[26] E. H. Aarts and J. H. Korst, "Simulated annealing," ISSUES, vol. 1, p. 16, 1988.

[27] A. G. Nikolaev and S. H. Jacobson, "Simulated annealing," in Handbook of metaheuristics, Springer, 2010, pp. 1-39.

[28] K. A. Dowsland and J. M. Thompson, "Simulated annealing," in Handbook of natural computing, Springer, 2012, pp. 1623-1655.

[29] F. Romeo and A. Sangiovanni-Vincentelli, "A theoretical framework for simulated annealing," Algorithmica, vol. 6, no. 1, pp. 302-345, 1991.

[30] M. Fleischer, "Simulated annealing: past, present, and future," in Simulation Conference Proceedings, 1995. Winter. IEEE, 1995, pp. 155-161.

[31] B. Suman and P. Kumar, "A survey of simulated annealing as a tool for single and multiobjective optimization," Journal of the operational research society, vol. 57, no. 10, pp. 1143-1160, 2006.

[32] P. J. Laarhoven, Theoretical and computational aspects of simulated annealing. Centrum voor wiskunde en informatica, 1988, vol. 51.

[33] V. Fabian, "Simulated annealing simulated," Computers \& Mathematics with Applications, vol. 33, no. 1-2, pp. 81-94, 1997.

[34] I. O. Bohachevsky, M. E. Johnson, and M. L. Stein, "Generalized simulated annealing for function optimization," Technometrics, vol. 28, no. 3, pp. 209-217, 1986.

[35] M. Locatelli, "Convergence and first hitting time of simulated annealing algorithms for continuous global optimization," Mathematical Methods of Operations Research, vol. 54, no. 2, pp. 171-199, 2001.

[36] C. J. Bélisle, H. E. Romeijn, and R. L. Smith, "Hit-and-run algorithms for generating multivariate distributions," Mathematics of Operations Research, vol. 18, no. 2, pp. 255-266, 1993.

[37] B. Hajek, "Cooling schedules for optimal annealing," Mathematics of operations research, vol. 13, no. 2, pp. 311-329, 1988.

[38] S. Anily and A. Federgruen, "Simulated annealing methods with general acceptance probabilities," Journal of Applied Probability, vol. 24, no. 03, pp. 657-667, 1987.

[39] D. Mitra, F. Romeo, and A. Sangiovanni-Vincentelli, "Convergence and finite-time behavior of simulated annealing," Advances in applied probability, vol. 18, no. 3, pp. 747-771, 1986.

[40] M. Lundy and A. Mees, "Convergence of an annealing algorithm," Mathematical programming, vol. 34, no. 1, pp. 111-124, 1986.

[41] B. Gidas, "Nonstationary markov chains and convergence of the annealing algorithm," Journal of Statistical Physics, vol. 39, no. 1-2, pp. 73-131, 1985.

[42] S. B. Gelfand and S. K. Mitter, "Analysis of simulated annealing for optimization," in Decision and Control, 1985 24th IEEE Conference on, vol. 24. IEEE, 1985, pp. 779-786. 